\title{
ALL POSITIVE INTEGERS ARE SUMS OF VALUES OF A QUADRATIC FUNCTION OF $x^{*}$
}

BY L. E. DICKSON

1. Introduction. Fermat stated that he was the first to discover the beautiful theorem that every integer $A \geqq 0$ is a sum of $m+2$ polygonal numbers

$$
p_{m+2}(x)=\frac{1}{2} m\left(x^{2}-x\right)+x
$$

of order $m+2$ (or $m+2$ sides), where $x$ is an integer $\geqq 0$. The cases $m=1$ and $m=2$ state that every $A$ is a sum of three triangular numbers $p_{3}(x)=\frac{1}{2} x(x+1)$, and also a sum of four squares $p_{4}(x)=x^{2}$.

Cauchy $\dagger$ was the first to publish a proof of Fermat's statement and showed that all but four of the polygonal numbers may be taken to be 0 or 1 .

In this paper and its sequel we shall give a complete solution of the following more general question.

Problem. Find every quadratic function $f(x)$ which takes integral values $\geqq 0$ for all integers $x \geqq 0$, such that every positive integer $A$ is a sum of $l$ of these values, where $l$ depends on $f(x)$, but not on $A$.

2. Lemma 1. A quadratic function of $x$ is an integer for every integer $x \geqq 0$ if and only if it is of the form

$$
f(x)=\frac{1}{2} m x^{2}+\frac{1}{2} n x+c, \quad m+n \text { even, }
$$

where $m, n$, and c are integers.

Consider $u x^{2}+v x+c$. By its values for $x=0,1$, and 2, $c, u+v$, and $4 u+2 v$ are integers. Subtract the double of the

* Presented to the Society, September 9, 1927.

† Oeuvres, (2), vol. 6, pp. 320-353. Pepin gave a modified proof, Atti dei Lincei, vol. 46 (1892-93), pp. 119-131. His proof requires a separate examination when $A<110 \mathrm{~m}$. For the simpler proof in $\$ 5$, the limit is $A<44 m+32$. 
second from the third. Hence $2 u$ is an integer $m$. Since $\frac{1}{2} m+v$ is an integer, $v$ is half an integer $n$.

3. Positive Quadratic Functions Representing 0 and 1. Let (2) be $\geqq 0$ for every integer $x \geqq 0$, whence $m>0, c \geqq 0$. Then

$$
f(x+1)-f(x)=m x+\frac{1}{2}(m+n)
$$

increases with $x$. Hence $f(x)$ does not represent every positive integer $A$. Thus $l>1$ in our problem, and a sum of two or more values of $f(x)$ must give $A=1$. Hence $f(u)=1$, $f(k)=0$ for certain integers $u \geqq 0, k \geqq 0$. We assume that $k$ has its least value. Then

$$
f(x)=f(x)-f(k)=\frac{1}{2}(x-k)[m(x+k)+n] .
$$

Since $f(u)=1$,

$$
n=s-m(u+k), \quad s=2 /(u-k),
$$

where $s$ is an integer. Thus $u-k= \pm 1$ or \pm 2 , and

$$
f(x)=\frac{1}{2}(x-k)[m(x-u)+s] .
$$

If $u-k= \pm 1$,

$$
f(x)=\frac{1}{2}(x-k)[m(x-k \mp 1) \pm 2]=p_{m+2}( \pm x \mp k) .
$$

If $u-k=2$,

$f(k+1)=\frac{1}{2}(1-m) \geqq 0$ gives $m=1, f(x)=p_{3}(x-k-1)$.

Finally, if $u-k=-2$, then $k \geqq 2$ and $f(k-1)=\frac{1}{2}(1-m)$ is zero, since it is not negative. But this contradicts the definition of $k$ as least.

THEOREM 1. The functions derived from (1) by replacing $x$ by $x-k$ or $k-x$ are the only quadratic functions of $x$ which are integers $\geqq 0$ for every integer $x \geqq 0$, and which take the values 0 and 1 for certain integers $x \geqq 0$.

The values of $p_{3}(-x)=\frac{1}{2}(x-1) x$ coincide with the triangular numbers. Hence if $m=1$, our problem is the same when $k \geqq 1$ as when $k=0$. This is evidently true also if $m=2$. Without loss of generality, we may then henceforth take $m>2$. 
4. Polynomials with an Excess. Let $f(x)$ have an integral value $\geqq 0$ for every integer $x \geqq 0$, and let one value be zero. Let $M_{s}(A)$ denote the maximum sum $\leqq A$ of $s$ values of $f(x)$, and write $E_{s}(A)$ for $A-M_{s}(A)$. In case $E_{s}(A)$ has a finite maximum $E_{s}$ for all integers $A \geqq 0$, every integer $A \geqq 0$ is a sum of $E_{s}$ numbers 0 or 1 and $s$ values of $f(x)$. Then $E_{s}$ is called the $s$-excess of $f(x)$. We shall drop the subscript 4 from $E_{4}$.

Let $\alpha, \beta, \gamma, \delta$ denote the four integral values $\geqq 0$ of $x$ and write

$$
a=\Sigma \alpha^{2}, \quad b=\Sigma \alpha .
$$

Take (2) as $f(x)$ and insert the four values of $x$. Thus

$$
A=\frac{1}{2} m a+\frac{1}{2} n b+4 c+r, \quad 0 \leqq r \leqq E,
$$

for a suitable integer $r$. Cauchy proved the following result.

LEMMA 2. If $a$ and $b$ are positive odd integers such that $b^{2}<4 a$ and

$$
b^{2}+2 b+4>3 a,
$$

equations (3) have solutions $\alpha, \beta, \gamma, \delta$ in integers $\geqq 0$.

Multiply (5) by $m$ and replace $m a$ by its value from (4). The resulting inequality follows from that obtained by suppressing $6 r$. Multiplication by $4 m$ now yields the equivalent inequality

$$
(2 m b+\tau)^{2}>U, \quad U=\tau^{2}+4 m(6 A-24 c-4 m),
$$

where $\tau=2 m+3 n$. This inequality holds if

$$
b>\left(U^{1 / 2}-\tau\right) /(2 m), \quad U \geqq 0 .
$$

To satisfy $b^{2}<4 a$, multiply by $m^{2}$ and replace $m a$ by its value from (4). The resulting condition evidently follows from that with $r$ replaced by $E$, and hence from

$$
(m b+2 n)^{2}<4 V, \quad V=n^{2}+m(2 A-8 c-2 E) .
$$

This inequality holds if

$$
b<\left(2 V^{1 / 2}-\quad\right) / m, \quad V \geqq 0,
$$


and if $m b+2 n \geqq 0$. For $n \geqq 0$, the latter evidently holds if $b>0$. For $n<0$, it holds by (7) if

$$
4 n-\tau+U^{1 / 2} \geqq 0
$$

and hence if

$$
3 A \geqq 12 c+2 m-2 n-n^{2} / m \quad(\text { if } n<0) .
$$

We desire that $b>0$. By (7), this will be true if $U^{1 / 2} \geqq \tau$. If $n<0$, this follows from (10). But if $n \geqq 0$, whence $\tau>0$, it holds if and only if $U \geqq \tau^{2}$, and hence if the quantity in the last parenthesis of $(6)$ is $\geqq 0$ :

$$
\left.A \geqq 4 c+\frac{2}{3} m \quad \text { (if } n \geqq 0\right) .
$$

There will be at least $d$ positive integers between the limits on $b$ stated in (7) and (9) if

$$
4 V^{1 / 2}-U^{1 / 2}>P, \quad P=2 m d-2 m+n .
$$

The left member is $\geqq 0$ if

$$
16 V \geqq U,
$$

and then (13) holds* if its square holds and hence if (15) $F \equiv(2 V+W)^{2}-V U>0, \quad 8 W \equiv U-P^{2}, P \geqq 0$.

By the minor conditions we shall mean $U \geqq 0, V \geqq 0$, the inequality (14), and (11) or (12). Since

(16) $16 V-U=3(2 m-n)^{2}+4 n^{2}+8 m(A-4 c-4 E)$,

it follows that (14) holds if

$$
A \geqq 4 c+4 E \text {. }
$$

The latter implies $V \geqq 0$. Evidently $U \geqq 0$ if

$$
A \geqq 4 c+\frac{2}{3} m \text {. }
$$

Hence the minor conditions all follow from (17) and (18) if $n \geqq 0$, but from these two and (11) if $n<0$. We shall speak of these as the reduced minor conditions.

* Automatically if $P<0$. 
5. Polygonal Numbers. We take (1) as the function (2). Thus $n=2-m, c=0$. By Table I, $E(2 m+3)=m-2$. Hence $E$ is not smaller than the value in

Theorem 2. For the function (1), $E_{4}=m-2$ if $m \geqq 3$.

The reduced minor conditions are all satisfied if $A \geqq 4 m$. Here (4) is $A=m g+b+r, g=\frac{1}{2}(a-b)$. If $b$ takes the odd values $\beta$ and $\beta+2$, while $r$ takes the values $0,1, \cdots$, $m-2$, the values of $b+r$ are $\beta+j(j=0,1, \cdots, m)$. These, with $j=m$ omitted, form a complete set of residues modulo $m$. Hence for any $A$, the preceding equation yields an integral value of $g$ and hence an odd integral value of $a$.

If there are at least $d=4$ integers between the limits for $b$, there will exist the desired two odd values for $b$. Then (6), (8), (13), and (15) give

$$
\begin{gathered}
U=24 m A-15 m^{2}-12 m+36, \quad V=2 m A-m^{2}+4, \\
P=5 m+2, \quad W=3 m A-5 m^{2}-4 m+4 \\
F=m^{2} A^{2}-44 m^{3} A-32 m^{2} A+34 m^{4}+44 m^{3}-56 m^{2} \\
-48 m>0 .
\end{gathered}
$$

Evidently $F>0$ if $A \geqq 44 m+32$.

Next, let $A<44 m+32$. Then $A<M \equiv 48 m+21$. In Table I the entries involving the same multiple of $m$, together with all intervening integers, will be said to form a block. We suppress $29 m+10-12$ and $45 m+10-13$. Down to $M$, the difference between any two consecutive numbers in any abridged block is now $\leqq 2$, whence $E(A) \leqq 1$ for every $A$ within a block. For every $A<M$ not within a block, $E(A) \leqq m-2$. This will follow if proved when $A+1$ is the first number of any abridged block. Then $A$ is the sum of $m-2$ and a number $t$ occurring explicitly in the abridged table except as follows. If $A=10 m+4,26 m+12,27 m+10$, or $28 m+12$, then $A=m-3+t$. If $A=6 m+3,21 m+6$, $28 m+7,30 m+11,34 m+11$, or $42 m+12$, then $A=m-4+t$; while, if $m=3, A$ is equal to the $A$ for the next smaller $m$. 
TABLE I.

\section{Sums of Four Polygonal Numbers}

$0-4, \quad m+2-5, \quad 2 m+4-6, \quad 3 m+3-7, \quad 4 m+5-8, \quad 5 m+7-8, \quad 6 m+4-9$, $7 m+6-9,8 m+8-10,9 m+7-10,10 m+5-11,11 m+7-9,11,12 m+8-12$, $13 m+8-12, \quad 14 m+10-12,15 m+6-9,11-13,16 m+8-13,17 m+10-13$, $18 m+9-14, \quad 19 m+11-14, \quad 20 m+10-14,21 m+7-13, \quad 15,22 m+9-15$, $23 m+11-15,24 m+10-16,25 m+11-13,15,16,26 m+13-16,27 m+11-16$, $28 m+8-11,13-17,29 m+10-12,15-17,30 m+12-17,31 m+11-17,32 m+$ $13-18,33 m+15-18,34 m+12-18,35 m+14-17,36 m+9-19,37 m+11-13$, $15-19,38 m+13-15,17-19,39 m+12-17,19,40 m+14-20,41 m+16-20$, $42 m+13-20,43 m+14-17,19,20,44 m+16-20,45 m+10-13,16-21$, $46 m+12-21,47 m+14-17,19-21,48 m+13-21,49 m+15-21,50 m+17-22$, $51 m+14-17, \quad 19-22,52 m+16-22,53 m+18-21,54 m+17-19,21,22$, $55 m+11-17,19-23,56 m+13-23,57 m+15-21,23,58 m+14-23,59 m+16$, $17,19-23,60 m+16-24,61 m+15-21,23,24,62 m+17-24,63 m+19-24$, $64 m+17-24,65 m+16-21,23,24,66 m+12-15,17-25,67 m+14-16,19-25$, $68 m+16,17,19-25,69 m+15-18,20,21,23-25,70 m+17-19,21-25$, $71 m+19-25,72 m+16-26,73 m+18-21,23-26,74 m+20-23,25,26$, $75 m+19-25, \quad 76 m+17-26, \quad 77 m+19-21, \quad 23-26, \quad 78 m+13-16, \quad 20-27$, $79 m+15-17,20-25,27,80 m+17,18,22-27,81 m+16-21,23-27,82 m+18-$ $27,83 m+19-25,27,84 m+17-28,85 m+19-21,23-28,86 m+21-28$, $87 m+19-25,27,28,88 m+18-22,24-28,89 m+20,21,23-28,90 m+20-23$, $25-28,91 m+14-17,20-25,27-29,92 m+16-18,22-29,93 m+18-21$, $23-29,94 m+17-29,95 m+19,20,22-2527-29,96 m+21-29,97 m+18-21$, $23-29,98 m+20-27,29,30,99 m+20-25,27-30,100 m+21-30,101 m+19-$ $21,23-29,102 m+21-30,103 m+22-25,27-30,104 m+22-30,105 m+15-18$, 24-29, 31, 106m +17-23, 25-31, 107m+19, 20, 22-25, 27-31, 108m+18-21, $24-31,109 m+20,21,23-29,31,110 m+22-31,111 m+19-25,27-31$, $112 m+21-32,113 m+23-29,31,32,114 m+22-27,29-32,115 m+20-22$, $24,25,27-32,116 m+22,23,25-32,117 m+23-29,31,32,118 m+23-32$, $119 m+22-25,27-32,120 m+16-19,21-33,121 m+18-20,23-29,31-33$, $122 m+20,21,25-33,123 m+19-25,27-33,124 m+21,22,25-33,125 m+23$, $25-29,31-33,126 m+20-31,33,127 m+22-25,27-33,128 m+24-26$, $28-34,129 m+23-29,31-34,130 m+21-23,25-27,29-34,131 m+23,24$, $28-33,132 m+24-34,133 m+23-29,31-34,134 m+25-34,135 m+22-24$, $27-33,136 m+17-20,24-35,137 m+19-21,26-29,31-35,138 m+21,22$, $25,26,28-35,139 m+20-23,27-33,35,140 m+22,23,26,27,29-35$, $141 m+23-29, \quad 31-35,142 m+21-31,33-35,143 m+23-25,27-33,35$, $144 m+25-36,145 m+24-29,31-36,146 m+22-27,29-36,147 m+24$, $25,27-33,35,36,148 m+24-27,29-36,149 m+25-29,31-36,150 m+25-36$, $151 m+23-25,27-33,35,36,152 m+25-36,153 m+18-21,27-29,31-37$, $154 m+20-22,26-37,155 m+22,23,28-33,35-37,156 m+21-37,157 m+$ $23-29,31-37,158 m+25-30,33-35,37,159 m+22-25,28-33,35-37,160 m$ $+24-37,161 m+26,28,29,31-37,162 m+25-27,29-38,163 m+23-25$, $27-33,35-38,164 m+25-27,30-38,165 m+26-28,31-37,166 m+26-38$, $167 m+28-33,35-38,168 m+24-26,29-31,33-38,169 m+26-29,31-37$, $170 m+28-38,171 m+19-22,27-33,35-39,172 m+21-23,26-39,173 m+23$, $24,28,29,31-37,39,174 m+22-31,33-35,37-39,175 m+24,25,27-33$, 
$35-39,176 m+26,29-39,177 m+23-26,28,29,31-37,39,178 m+25-27$, 29-39, 179m + 27, 31-33, 35-39, 180m+26-40, 181m+24-29, 31-37, 39, 40, $182 m+26-40,183 m+27-33,35-40, \quad 184 m+27-40, \quad 185 m+29, \quad 31-37$, $39,40,186 m+25-40,187 m+27-33,35-40,188 m+29,30,32-40,189 m$ $+27-29,31-37,39,40,190 m+20-23,29-31,33-35,37-41,191 m+22-24$, $28-33,35-41,192 m+24-41,193 m+23-26,28,29,31-37,39-41,194 m+25$, $26,30-32,34-39,41,195 m+27,29-33,35-41,196 m+24-27,29-41,197 m$ $+26-28,31-37,39-41,198 m+28-41,199 m+27,28,30-32,35,36$.

If $A=15 m+5$ or $46 m+11$, then $A=m-5+t$; while, if $m=3$ or $4, A$ is $\leqq$ the $A$ for the next smaller $m$. Finally, if $A=36 m+8$, then $A=m-6+t$; while, if $m=3,4$, or 5 , $A=34 m+14,16$, or 18 , which belong to an earlier block. This completes the proof of Theorem 2 .

By that theorem, every integer $A \geqq 0$ is a sum of $m+2$ polygonal numbers. Hence $E_{s}=0$ if $s \geqq m+2$. Next, let $4 \leqq s<m+2$. If a sum by $s$ of the polygonal numbers $0,1, m+2,3 m+3, \cdots$ is $\leqq 2 m+3$, at most one summand is $m+2$, whence the maximum such sum is $m+2+s-1$. Hence $E_{s}(2 m+3)=m-s+2$. By Theorem 2, $A$ is a sum of four polygonal numbers and $m-2$ numbers 0 or 1 . Regard $s-4$ of the latter as polygonal numbers. Hence $A$ is a sum of $s$ polygonal numbers and $m-s+2$ numbers 0 or 1 . All of these facts prove the following theorem.

THEOREM 3. For the function (1), $E_{s}=0$ if $s \geqq m+2$, while $E_{s}=m-s+2$ if $4 \leqq s \leqq m+2$.

In the second case, $s+E_{s}=m+2$, so that the use of 5 or more polygonal numbers $>1$ yields no gain (but rather a loss) over the use of only four.

6. Deductions from Table I. We extended our table beyond the limit $48 m+21$ required for the proof of Theorem 2 in order to deduce interesting facts concerning $E(A)$ for func. tion (1), which are essential to the sequel.

LEMMA 3. For $54 m+17 \leqq A<74 m+28, E(A) \leqq m-6$ if $m \geqq 7, E(A) \leqq 1$ if $m=5$ or 6 .

From Table I we suppress $67 m+14-16$. Since the difference between any two consecutive numbers in any abridged block is now $\leqq 2, E(A) \leqq 1$, for every $A$ within a 
block. Let $f$ be the term free of $m$ in the leader $q m+f$ of any abridged block. First, let $m=5$. For $q=56, \cdots, 74$, we find that $f+4$ is the term free of $m$ in a number of the preceding abridged block. Hence $q m+f-1$ is the sum of $m-5$ and the number $(q-1) m+f+4$ in the abridged table. Finally, the $E$ of $55 m+10=53 m+20$ is 1 . Second, let $m \geqq 6$. Except for $q=55,56,62,70, f+5$ is the term free of $m$ in a number of the preceding abridged block, whence $E(A) \leqq m-6$. For the four $q$ 's, we use $f+6$ if $m>6$. If $m=6, \quad 56 m+12=55 m+18, \quad 62 m+16=61 m+22, \quad 70 m+16$ $=69 m+22$, which fall within preceding blocks, while $55 m+1<54 m+17$.

Lemma 4 . For $74 m+20 \leqq A \leqq 199 m+37, E(A) \leqq 1$ if $m=7, E(A) \leqq m-7$ if $m \geqq 8$, except that $E(80 m+21)=m-6$ if $m=8$ or 9 .

From each block we suppress all entries down to and including the last entry which differs by 3 or more from the next entry. As in Lemma 3, $f+6$ succeeds except for $q=80$, $106,156,158,169,195$. For $m=7, a=80 m+21$ equals $79 m+28$, whose $E$ is 1 . For $m \geqq 10$, we restore the previously excluded $80 m+17-18$ and then have the permissible value $E(a)=3$ and $80 m+16=m-7+79 m+23$. But for $m=8$ or 9 , $80 m+18 \leqq 79 m+27=a^{\prime}$, and the full table includes no number numerically between $a^{\prime}$ and $a$, whence $E(a)=m-6$.

For $q=106,169,195$, we may use $f+6$ after suppressing $106 m+17-23,169 m+26-29$, and $195 m+27$.

For $m \geqq 8, b=156 m+20=m-8+155 m+28$. But if $m=7$, $b=155 m+27$, which was treated under $q=155$.

Finally, let $q=158$. If $m \geqq 9$ we restore the previously excluded $158 m+25-30$, noting that $E(158 m+32)=2$ is a permissible value. Then $158 m+24=m-7+t$, where $t=157 m+31$ is in the table. If $m=7$ or 8 , the latter result is applicable since the missing $158 m+31-32$ are equal to two of $159 m+23-25$.

Assistance was provided by the Carnegie Institution for the construction of Table I and checking results by it.

The University of Chicago 